\title{
TEMPERATURE AND THERMAL STRESSES DISTRIBUTIONS IN A DRY FRICTION CLUTCH
}

\author{
KATARZYNA TOPCZEWSKA \\ Bialystok University of Technology, Bialystok, Poland \\ e-mail: k.topczewska@pb.edu.pl \\ Josef SChLATTMANN \\ Hamburg University of Technology, Hamburg, Germany \\ OdAy IbraheEm AbDullah \\ University of Baghdad, Baghdad, Iraq, and \\ Hamburg University of Technology, Hamburg, Germany
}

\begin{abstract}
The aim of this study is to develop an analytical model to determine temperature and thermal stresses distributions in a single plate clutch during the slipping phase. Proposed expressions enable calculation of temperature and thermal stresses on the contact surface as well as at any depth inside the friction material and at any instant during the slipping period. It was investigated how the maximum temperature on the friction surface varies with the thickness of the friction facings of the clutch disc and the intensity of convective cooling.
\end{abstract}

Keywords: friction, clutch, temperature, thermal stresses

\section{Introduction}

The most critical period during engagement of the clutch is the sliding phase, when a high amount of heat is generated on the contact surfaces due to friction. In order to prevent failure of the clutch system, it is necessary to estimate the variation of temperature and how it depends on operating conditions such as load, degree of convective cooling, material properties and dimensions of the friction elements.

Computation of the temperature field generated during the heating phase (sliding period) for the friction clutch using analytical and numerical approaches have been developed by many researchers (Abdullah and Schlattmann, 2012; Grzelczyk and Awrejcewicz, 2012; Faidh-Allah, 2017). In the proposed analytical models, the following were assumed: one-dimensional heat flow, omission of convective heat exchange, uniform distribution of frictional heat flux on contact surfaces, replacement of real friction elements with semi-infinite bodies and constant, linear increase or parabolic increase of friction torque (Newcomb, 1960, 1961). The developed numerical models based on the finite element method allowed taking into account convective heat exchange to the surrounding environment, and finite dimensions of friction elements and also different torque profiles. In the paper (Abdullah and Schlattmann, 2014), the change of torque during the heating phase of the clutch engagement was described by a time-dependent function which was universal for different engagement modes. The exact, fully-analytical formula to calculate temperature on the friction surface of a clutch disc during engagement with the friction power described by the mentioned function was obtained in the reference (Kuciej and Topczewska, 2019). This model was developed based on the solution to the boundary-value problem of heat conduction formulated for a tribosystem consising of two semi-infinite bodies. Omission of real 
dimensions of friction elements and convective cooling is reasonable when the operating conditions are such that the generated heat affects only a thin layer of the material in the immediate vicinity of the friction surface. However, these factors are important for long-duration slipping processes under heavy loads in systems with well-ventilated clutch discs or cases with repetitive engagements.

In this study, a new analytical model to calculate the distribution of temperature in friction facings of the clutch disc was developed. The analytical model is built based on the two-dimensional boundary-value problem of heat conduction formulated for a rectangular strip with finite thickness. Additionally, the convective heat exchange with the surrounding environment on lateral surfaces of frictional elements was taken into account. It should be noted that the investigation of wear mechanism in automotive clutches and brakes (Al-Alawi et al., 2007) is necessary, but in this work the wear effect is ignored because the slipping period is very short and the analysis is achieved only for a single engagement. Therefore, in this case, wear on the contact surfaces is neglected.

\section{Mathematical model}

The typical design of the single-plate clutch system consists of a flywheel, pressure plate and a clutch disc. The clutch disc has two effective frictional facings attached as it is shown in Fig. 1.

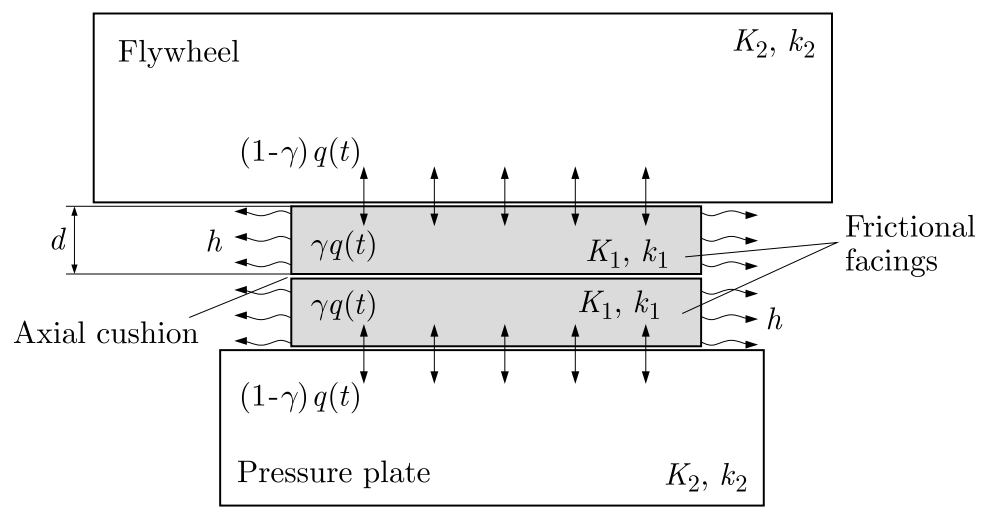

Fig. 1. The flow of heat generated on the friction surfaces of a single-plate clutch

During the engagement period, the elements of the clutch system are pressed to each other, and the friction process occurs on the contact surfaces till the end of slipping phase. Assuming uniform wear, the moment of friction force changes with slipping time can be written as in the following (Abdullah and Schlattmann, 2014)

$$
M(t)=M_{0} M^{*}(t) \quad M^{*}(t)=\frac{t}{t_{s}}\left[2-\left(\frac{t}{t_{s}}\right)^{\alpha}\right] \quad 0 \leqslant t \leqslant t_{s} \quad 0 \leqslant \alpha \leqslant 1
$$

where $\alpha$ is the index parameter. The initial problem of motion for the considered system is

$$
I \frac{d \omega(t)}{d t}=-M(t) \quad 0<t \leqslant t_{s} \quad \omega(0)=\omega_{0}
$$

where $I=\left(1 / I_{1}+1 / I_{2}\right)^{-1}, \omega$ - relative angular velocity, $I_{1}$ - moment of inertia of the input, $I_{2}$ - moment of inertia of the output, $t_{s}$ - slipping time.

The solution to equation of motion (2.2) with account of Eq. (2.1) gives us

$$
\omega(t)=\omega_{0} \omega^{*}(t) \quad \omega^{*}(t)=1-\frac{1}{\alpha+1}\left(\frac{t}{t_{s}}\right)^{2}\left[\alpha+2-\left(\frac{t}{t_{s}}\right)^{\alpha}\right] \quad 0 \leqslant t \leqslant t_{s}
$$


and the time required to couple the two inertias was calculated from the condition $\omega\left(t_{s}\right)=0$

$$
t_{s}=\frac{(\alpha+2) I \omega_{0}}{(\alpha+1) M_{0}}
$$

The thermal load applied to the contact surface is directly proportional to the specific power of friction (Abdullah and Schlattmann, 2014)

$$
q(t)=q_{0} q^{*}(t) \quad q_{0}=\frac{M_{0} \omega_{0}}{A_{a}} \quad A_{a}=2 \pi\left(r_{e}^{2}-r_{i}^{2}\right) \quad 0 \leqslant t \leqslant t_{s}
$$

and

$$
q^{*}(t)=\frac{1}{\alpha+1}\left[2(\alpha+1) \frac{t}{t_{s}}-2(\alpha+2)\left(\frac{t}{t_{s}}\right)^{3}-(\alpha+1)\left(\frac{t}{t_{s}}\right)^{\alpha+1}+(\alpha+4)\left(\frac{t}{t_{s}}\right)^{\alpha+3}-\left(\frac{t}{t_{s}}\right)^{2 \alpha+3}\right]
$$

where $A_{a}$ is the total nominal area of contact, $r_{e}, r_{i}$ - outer and inner radius of the disc clutch. Based on Eq. (2.6), one can establish the total heat generated in the friction clutch due to friction during the heating phase

$$
Q=A_{a} \int_{0}^{t_{s}} q(t) d t=\frac{I \omega_{0}^{2}}{2}
$$

It should be noted that the amount of heat dissipated in the clutch system does not depend on the value of $\alpha$ parameter.

In order to find the temperature field caused by dissipation of heat in Eq. (2.7), one should formulate the thermal friction problem accurately. Owing to symmetry of the friction clutch system (Fig. 1), the temperature field in the friction facing of the clutch disc is uniformly distributed in the circumferential direction. Therefore, the complexity of the thermal problem from three dimensions can be reduced to a two-dimensional strip as shown in Fig. 2.

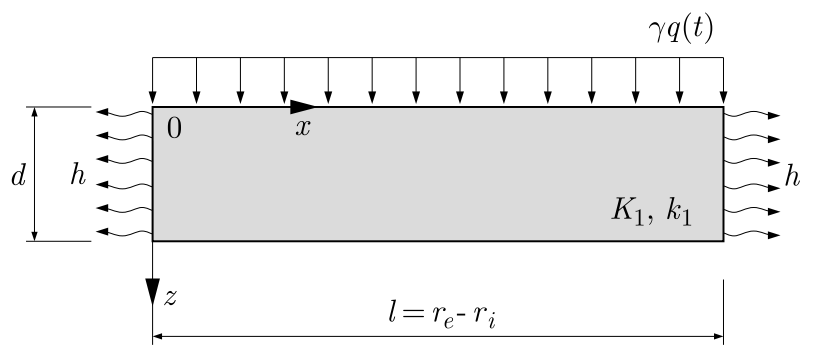

Fig. 2. Scheme of the friction element

The transient temperature distribution $T(x, z, t)$ within the strip will be found from the solution of the two-dimensional heat conduction equation

$$
\frac{\partial^{2} T(x, z, t)}{\partial x^{2}}+\frac{\partial^{2} T(x, z, t)}{\partial z^{2}}=\frac{1}{k_{1}} \frac{\partial T(x, z, t)}{\partial t} \quad \begin{aligned}
& 0<x<l \\
& 0<z<d \\
& 0<t \leqslant t_{s}
\end{aligned}
$$

where $k_{1}=K_{1} /\left(\rho_{1} c_{1}\right)$ - thermal diffusivity of the friction material.

On the upper edge of the strip $z=0$, the frictional thermal load $q(t)$ from Eq. (2.6) is applied

$$
\left.K_{1} \frac{\partial T(x, z, t)}{\partial z}\right|_{z=0}=-\gamma q(t) \quad 0<x<l \quad 0<t \leqslant t_{s}
$$


where the heat partition coefficient $\gamma$ which establishes what part of generated heat is absorbed by this particular element was calculated from the following formula (Charron, 1943)

$$
\gamma=\frac{\sqrt{K_{1} \rho_{1} c_{1}}}{\sqrt{K_{1} \rho_{1} c_{1}}+\sqrt{K_{2} \rho_{2} c_{2}}}
$$

where $K$ - thermal conductivity, $\rho$ - density and $c$ - specific heat capacity of the friction facing material. The symbols with subscripts 1 refer to the friction material and 2 refer to the second element of the friction pair (flywheel or pressure plate) materials.

In well-ventilated clutch systems, intensive convective heat exchange with environment takes place on the exposed surfaces of the friction elements. Thus, on the lateral edges of the strip $x=0$ and $x=l$ the Newton law of cooling is applied

$$
\begin{array}{ll}
\left.K_{1} \frac{\partial T(x, z, t)}{\partial x}\right|_{x=0}=h\left[T(0, z, t)-T_{a}\right] & \\
\left.K_{1} \frac{\partial T(x, z, t)}{\partial x}\right|_{x=l}=h\left[T_{a}-T(l, z, t)\right] & 0<z<d \quad 0<t \leqslant t_{s}
\end{array}
$$

where $h$ is a constant heat transfer coefficient. Additionally, the boundary condition of thermal insulation on the bottom edge of the strip $z=d$ is

$$
\left.K_{1} \frac{\partial T(x, z, t)}{\partial z}\right|_{z=d}=0 \quad 0<x<l \quad 0<t \leqslant t_{s}
$$

and the initial condition:

$$
T(x, z, 0)=T_{a} \quad 0 \leqslant x \leqslant l \quad 0 \leqslant z \leqslant d
$$

where $T_{a}$ is ambient temperature included to the problem.

In order to find the temperature field, equation of heat conduction (2.8) was transformed. This was reduced to a one-dimensional form (Luikov, 1968) by including the conditions of convective cooling (2.11) in it

$$
\frac{\partial^{2} T(z, t)}{\partial z^{2}}-\frac{2 h^{*}}{K_{1} l}\left[T(z, t)-T_{a}\right]=\frac{1}{k_{1}} \frac{\partial T(z, t)}{\partial t} \quad 0<z<d \quad 0<t \leqslant t_{s}
$$

where

$$
T(z, t)=\frac{1}{l} \int_{0}^{l} T(x, z, t) d x \quad h^{*}=\left(\frac{1}{h}+\frac{l}{2 K_{1}}\right)^{-1}
$$

Introducing dimensionless variables and parameters

$$
\begin{array}{lrr}
\Theta(\zeta, \tau) \mathrm{e}^{-\mathrm{Bi} \tau}=\frac{T(z, t)-T_{a}}{T_{0}} & T_{0}=\frac{q_{0} d}{K_{1}} & \zeta=\frac{z}{d} \\
\tau=\frac{k_{1} t}{d^{2}} & \tau_{s}=\frac{k_{1} t_{s}}{d^{2}} & \mathrm{Bi}=\frac{2 h^{*} d^{2}}{K_{1} l}
\end{array}
$$

equation (2.14) with boundary (2.9), (2.12) and initial (2.13) conditions were transformed into the following forms 


$$
\begin{aligned}
& \frac{\partial^{2} \Theta(\zeta, \tau)}{\partial \zeta^{2}}=\frac{\partial \Theta(\zeta, \tau)}{\partial \tau} \quad 0<\zeta<1 \quad 0<\tau \leqslant \tau_{s} \\
& \left.\frac{\partial \Theta(\zeta, \tau)}{\partial \zeta}\right|_{\zeta=0}=-q^{*}(\tau) \mathrm{e}^{\mathrm{Bi} \tau} \quad 0<\tau \leqslant \tau_{s} \\
& \left.\frac{\partial \Theta(\zeta, \tau)}{\partial \zeta}\right|_{\zeta=1}=0 \quad 0<\tau \leqslant \tau_{s} \\
& \Theta(\zeta, 0)=0 \quad 0 \leqslant \zeta \leqslant 1
\end{aligned}
$$

Solution of the problem of heat conduction Eqs. (2.17) was found based on Duhamel's theorem (Ozisik, 1993)

$$
\Theta(\zeta, \tau)=-\int_{0}^{\tau} q^{*}(s) \mathrm{e}^{\mathrm{Bis}} \frac{\partial}{\partial \tau} \Theta_{0}(\zeta, \tau-s) d s \quad 0 \leqslant \tau \leqslant \tau_{s}
$$

where (Hetnarski and Eslami, 2009)

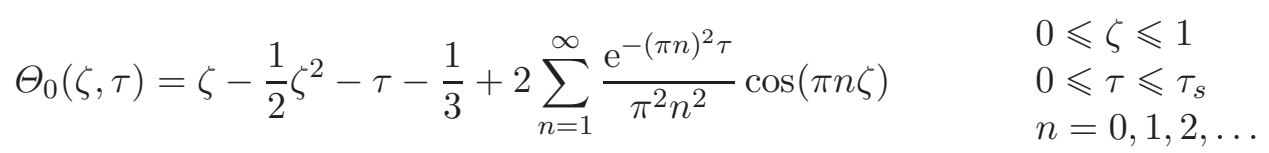

The differentiating function $\Theta_{0}(\zeta, \tau-s)(2.19)$ with respect to the dimensionless time $\tau$ and substitution the result to Equation (2.18) gives

$$
\Theta(\zeta, \tau)=J_{0}(\tau)+2 \sum_{n=1}^{\infty} \mathrm{e}^{-(\pi n)^{2} \tau} J(\tau) \cos (\pi n \zeta) \quad 0 \leqslant \tau \leqslant \tau_{s}
$$

where

$$
J(\tau)=\int_{0}^{\tau} q^{*}(s) \mathrm{e}^{\mathrm{Bi}_{n} s} d s \quad \mathrm{Bi}_{n}=\mathrm{Bi}+\pi^{2} n^{2} \quad n=0,1,2, \ldots
$$

Integral (2.21) with account of function (2.6) can be written in the form

$$
\begin{aligned}
& J(\tau)=\frac{1}{\alpha+1}\left[2(\alpha+1) J_{1}(\tau)-2(\alpha+2) J_{3}(\tau)-(\alpha+1) J_{\alpha+1}(\tau)\right. \\
& \left.\quad+(\alpha+4) J_{\alpha+3}(\tau)-J_{2 \alpha+3}(\tau)\right] \quad 0 \leqslant \tau \leqslant \tau_{s} \quad 0 \leqslant \alpha \leqslant 1
\end{aligned}
$$

where

$$
J_{n}^{(i)}(\tau)=\int_{0}^{\tau}\left(\frac{s}{\tau_{s}}\right)^{i} \mathrm{e}^{\mathrm{Bi}_{n} s} d s \quad \begin{aligned}
& i=1,3, \alpha+1, \beta+3 \\
& \quad 0 \leqslant \alpha \leqslant 1 \\
& \beta=\alpha, 2 \alpha
\end{aligned}
$$

Integrating by parts (Gradshteyn and Ryzhik, 2014), the following can be obtained

$$
\begin{aligned}
& J_{n}^{(1)}(\tau)=\frac{1}{\tau_{s} \mathrm{Bi}_{n}{ }^{2}}\left[\mathrm{e}^{\mathrm{Bi}_{n} \tau}\left(\mathrm{Bi}_{n} \tau-1\right)+1\right] \\
& J_{n}^{(3)}(\tau)=6\left(\frac{\tau}{\tau_{s}}\right)^{3} \frac{\mathrm{e}^{\mathrm{Bi}_{n} \tau}}{\mathrm{Bi}_{n^{4}}}\left\{\frac{\mathrm{Bi}_{n^{3}}}{6}+\frac{1}{\tau^{3}}\left[\mathrm{e}^{-\mathrm{Bi}_{n} \tau}-1+\mathrm{Bi}_{n} \tau\left(1-\frac{\mathrm{Bi}_{n} \tau}{2}\right)\right]\right\} \\
& J_{n}^{(\alpha+1)}(\tau)=(\alpha+1)\left(\frac{\tau}{\tau_{s}}\right)^{\alpha+1} \frac{\mathrm{e}^{\mathrm{Bi}_{n} \tau}}{\mathrm{Bi}_{n}}\left(\frac{1}{\alpha+1}-\frac{1}{\tau^{\alpha+1} \mathrm{e}^{\mathrm{Bi}_{n} \tau}} I_{\alpha, n}(\tau)\right) \\
& J_{n}^{(\beta+3)}(\tau)=(\beta+3)\left(\frac{\tau}{\tau_{s}}\right)^{\beta+3} \frac{\mathrm{e}^{\mathrm{Bi}_{n} \tau}}{\mathrm{Bi}_{n}{ }^{3}}\left[\frac{\beta+2}{\tau^{2}}\left(1-\frac{\beta+1}{\tau^{\beta+1} \mathrm{e}^{\mathrm{Bi}_{n} \tau}} I_{\beta, n}(\tau)\right)+\frac{\mathrm{Bi}_{n}}{\beta+3}-\frac{\mathrm{Bi}_{n}}{\tau}\right] \\
& I_{\beta, n}(\tau)=\int_{0}^{\tau} s^{\beta} \mathrm{e}^{\mathrm{Bi}_{n} s} d s \quad \beta=\alpha, 2 \alpha \quad 0 \leqslant \alpha \leqslant 1
\end{aligned}
$$


Owing to the lack of an exact analytical solution to the integral (last Eqs. (2.24)), it had to be computed numerically by means of an adaptive quadrature method.

Heterogeneity of the obtained above temperature field $T(z, t)$ causes generation of thermal stresses. These stresses were found based on the model of thermal bending of a thick plate with unfixed ends (Noda et al., 2000). In accordance to this model, the normal components of the stress tensor $\boldsymbol{\sigma}$ can be computed from the following relations

$$
\begin{aligned}
& \sigma_{x}(z, t)=\sigma_{y}(z, t) \equiv \sigma(z, t) \quad \sigma_{z}(z, t)=0 \\
& \sigma(z, t)=\sigma_{0} \sigma^{*}(\zeta, \tau) \quad \sigma_{0}=\frac{\alpha_{1} E_{1} T_{0}}{1-\nu_{1}} \quad 0 \leqslant z \leqslant d \quad 0 \leqslant t \leqslant t_{s} \\
& \sigma^{*}(\zeta, \tau)=\varepsilon^{*}(\zeta, \tau)-T^{*}(\zeta, \tau) \quad 0 \leqslant \zeta \leqslant 1 \quad 0 \leqslant \tau \leqslant \tau_{s} \\
& \varepsilon^{*}(\zeta, \tau)=(4-6 \zeta) N(\tau)+6(2 \zeta-1) M(\tau) \\
& N(\tau)=\int_{0}^{1} T^{*}(\zeta, \tau) d \zeta \quad M(\tau)=\int_{0}^{1} \zeta T^{*}(\zeta, \tau) d \zeta
\end{aligned}
$$

where $\alpha_{1}$ - Young's modulus [MPa], $\alpha_{1}$ - coefficient of the linear thermal expansion $\left[\mathrm{K}^{-1}\right]$, $\nu_{1}$ - Poisson's ratio of the friction material and $T_{0}$ is already determined by Eq. (2.16).

\section{Results and discussion}

Based on the obtained results from the newly developed analytical solution to the thermal problem of the friction clutch, the temperature and thermal stresses distributions in the friction facings of the clutch disc during a single engagement were found. The input parameters used for the calculations are presented in Table 1. Based on the input parameters, firstly we calculated the total nominal contact area $A_{a}=0.023 \mathrm{~m}^{2}$, nominal value of specific friction power $q_{0}=3.78 \cdot 10^{6} \mathrm{~W} / \mathrm{m}^{2}(2.6)$, total heat generated $Q=1.67 \cdot 10^{4} \mathrm{~J}(2.7)$ and Biot number $\mathrm{Bi}=0.028$ (2.16).

Table 1. Input parameters (Abdullah and Schlattmann, 2014; Abdullah et al., 2015)

\begin{tabular}{|l|c|}
\hline \multicolumn{1}{|c|}{ Parameter symbol [unit] } & Value \\
\hline \hline Coefficient of heat exchange with environment $h\left[\mathrm{~W} /\left(\mathrm{m}^{2} \mathrm{~K}\right)\right]$ & 40.89 \\
\hline Thickness of friction element $d[\mathrm{~mm}]$ & 3 \\
\hline Thermal conductivity of friction material $K_{1}[\mathrm{Wm} / \mathrm{K}]$ & 0.6 \\
\hline Thermal conductivity of flywheel and pressure plate $K_{2}[\mathrm{Wm} / \mathrm{K}]$ & 42 \\
\hline Thermal diffusivity of friction material $k_{1}\left[\mathrm{~m}^{2} / \mathrm{s}\right]$ & $7.16 \cdot 10^{-7}$ \\
\hline Thermal diffusivity of flywheel and pressure plate $k_{2}\left[\mathrm{~m}^{2} / \mathrm{s}\right]$ & $1.2 \cdot 10^{-5}$ \\
\hline Young's modulus of friction material $E_{1}[\mathrm{MPa}]$ & 300 \\
\hline Coefficient of linear thermal expansion of friction material $\alpha_{1}\left[\mathrm{~K}^{-1}\right]$ & $12.1 \cdot 10^{-6}$ \\
\hline Poisson's ratio of friction material $\nu_{1}[-]$ & 0.25 \\
\hline Internal radius of clutch disc $r_{i}[\mathrm{~mm}]$ & 62.98 \\
\hline External radius of clutch disc $r_{e}[\mathrm{~mm}]$ & 87.21 \\
\hline Effective moment of inertia $I\left[\mathrm{~kg} \mathrm{~m}{ }^{2}\right]$ & 0.8333 \\
\hline Nominal moment of friction $M_{0}[\mathrm{~N} \mathrm{~m}]$ & 432 \\
\hline Initial relative angular velocity $\omega_{0}[\mathrm{rad} / \mathrm{s}]$ & 200 \\
\hline Initial temperature $T_{a}[\mathrm{~K}]$ & 300 \\
\hline
\end{tabular}

The variations of temperature $T(z, t)$ and the corresponding normal thermal stresses $\sigma(z, t)$ on the friction surface $z=0 \mathrm{~mm}$ and inside the element for a few distances from this surface 

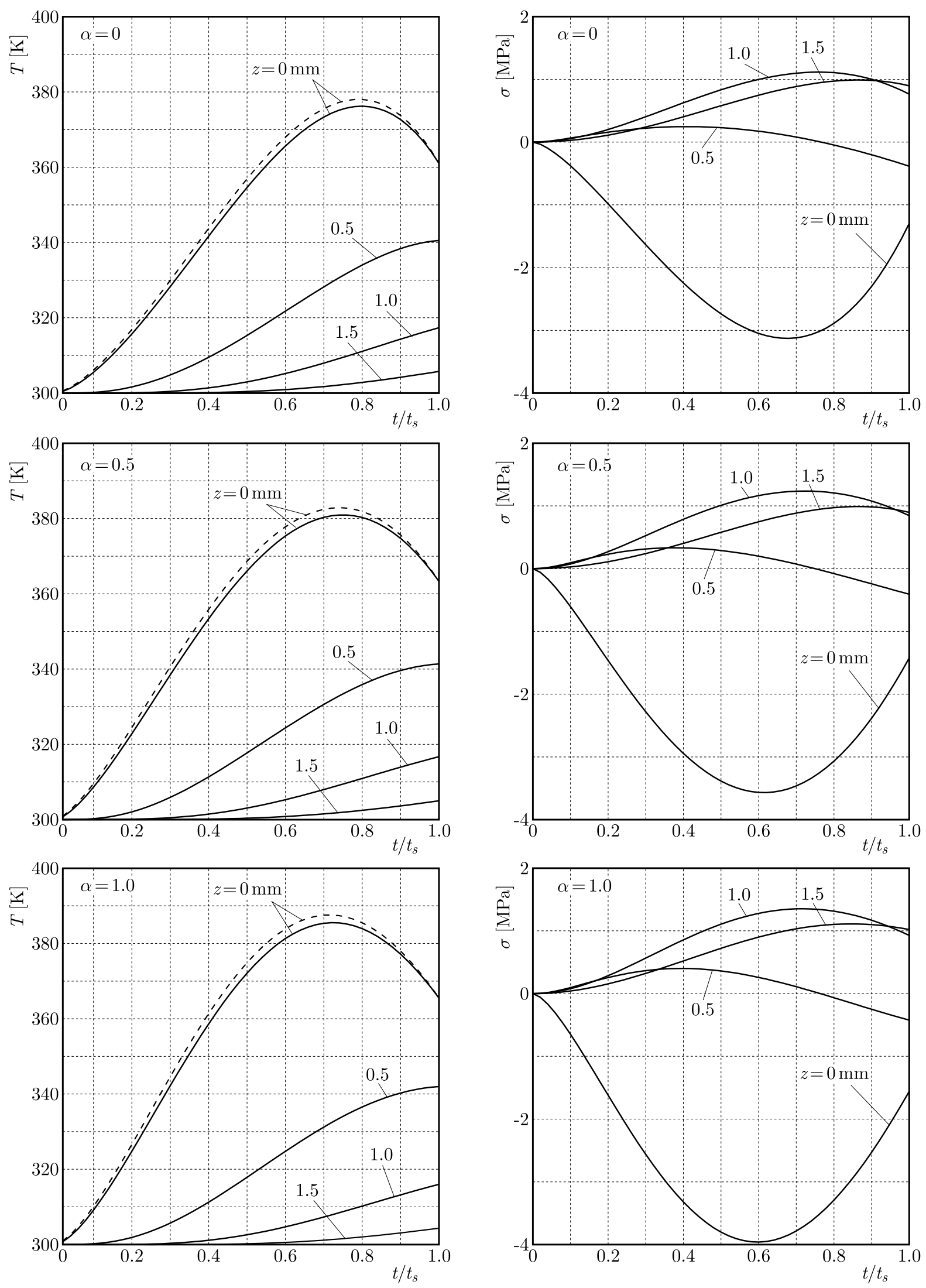

Fig. 3. Evolutions of temperature $T(z, t)$ and corresponding normal thermal stresses $\sigma(z, t)$ in the friction surface $z=0 \mathrm{~mm}$ and inside the friction material at different depths $z$. Solid lines - results obtained from the developed analytical solution, dashed lines - corresponding results based on solution (3.2) from the reference (Kuciej and Topczewska, 2019) 
are presented in Fig. 3. Solid lines illustrate results based on the developed analytical solution for the above input parameters (Table 1) and dashed lines illustrate the corresponding data obtained from the solution presented in the reference (Kuciej and Topczewska, 2019) as in the following form

$$
T(t)=T_{a}+T_{0} T^{*}(\tau) \quad 0 \leqslant \tau \leqslant \tau_{s}
$$

and

$$
\begin{aligned}
& T^{*}(\tau)=\gamma \sqrt{\tau}\left(\frac{\tau}{\tau_{s}}\right)\left\{\frac{1}{\sqrt{\pi}}\left[\frac{8}{3}-\frac{64(\alpha+2)}{35(\alpha+1)}\left(\frac{\tau}{\tau_{s}}\right)^{2}\right]\right. \\
&\left.-\left(\frac{\tau}{\tau_{s}}\right)^{\alpha}\left[\frac{\Gamma(\alpha+2)}{\Gamma(\alpha+2.5)}-\frac{\Gamma(\alpha+5)}{(\alpha+1) \Gamma(\alpha+4.5)}\left(\frac{\tau}{\tau_{s}}\right)^{2}+\frac{\Gamma(2 \alpha+4)}{(\alpha+1) \Gamma(2 \alpha+4.5)}\left(\frac{\tau}{\tau_{s}}\right)^{\alpha+2}\right]\right\}
\end{aligned}
$$

where $\Gamma(x)$ is the gamma function. The above relation allow one to calculate the temperature on the friction surface of the clutch disc during engagement for the considered specific friction power (2.5) and (2.6). This model (3.2) was developed from the solution to the one-dimensional boundary-value problem of heat conduction formulated for a tribosystem consisting of two semiinfinite bodies (Kuciej and Topczewska, 2019). Therefore, it does not take into account the influence of thickness of the frictional facings and convective cooling on its lateral surfaces. It can be seen in Fig. 3 that the evolutions of temperature in the friction surface obtained from both solutions are close to each other. The temperature calculated for semi-spaces (3.2) is slightly higher, the differences between these results do not exceed $\Delta T=5 \mathrm{~K}$ for all considered cases.

The effect of the index parameter $\alpha$ on the maximum temperature was investigated under different working conditions as shown in Fig. 3. Three different values of the index parameter $\alpha$ were assumed in these calculations. The lowest temperatures and absolute values of thermal stresses are achieved in the case of $\alpha=0$, when the friction process is the longest - it takes $t_{s}=0.77 \mathrm{~s}$. When the value of $\alpha$ parameter increases, it causes reduction of the sliding time to $t_{s}=0.64 \mathrm{~s}$ for $\alpha=0.5$ and $t_{s}=0.58 \mathrm{~s}$ for $\alpha=1$. This also causes an increase in achieved temperatures and thermal stresses because the total heat generated during all considered processes is the same (2.7). The maximum values of temperature are found to be $T_{\max }=376 \mathrm{~K}, 381 \mathrm{~K}$, $386 \mathrm{~K}$ and the absolute stresses $\left|\sigma_{\max }\right|=3.13 \mathrm{MPa}, 3.57 \mathrm{MPa}, 3.96 \mathrm{MPa}$, corresponding to $\alpha=0$, 0.5 and 1 , respectively. It was found that the thermal stresses are compressive during the whole sliding process at the level $z=0 \mathrm{~mm}$. The values of temperature and thermal stresses decreased while going deeper from the contact surface $(z=0)$ through the thickness. At the same time, inside the frictional facing, it was found that the thermal stresses are tensile, and their highest values occurred at about $1 \mathrm{~mm}$ in depth. At the distance $z=1.5 \mathrm{~mm}$, the temperature rise during the process is almost unnoticeable.

On the left side of Fig. 4, one can be notice the dependency of the maximum temperature on thickness of the frictional facing $d$ in the range of $0.5 \leqslant d \leqslant 3 \mathrm{~mm}$ for different parameter values $\alpha$. For the thinnest element $d=0.5 \mathrm{~mm}$, the temperature attains the highest maximum value $T_{\max }=404 \mathrm{~K}$, which is almost equal for all cases. When increasing the thickness $d$, the maximum temperature drops and then stabilizes in the range of $a \leqslant d \leqslant 3$, where $a=1.29 \mathrm{~mm}$, $1.17 \mathrm{~mm}, 1.11 \mathrm{~mm}$ for $\alpha=0,0.5$ and 1 , respectively. This parameter is a theoretical distance from the heated surface in which the temperature achieves $5 \%$ of the maximum value, and it is called the effective depth of heat penetration (Yevtushenko et al., 2019). It should be noted that increasing of the frictional facing thickness $d$ causes more intensive convective heat exchange due to enlargement of the lateral surfaces area. Therefore, the Biot number also varies from $\mathrm{Bi}=7.7 \cdot 10^{-4}$ for $d=0.5 \mathrm{~mm}$ to $\mathrm{Bi}=0.028$ for $d=3 \mathrm{~mm}$. However, these small values of the Biot number do not significantly affect the temperature in the considered processes. It can be observed in the right side of Fig. 4 that the maximum temperature monotonically and linearly decreased to $T_{\max }=367 \mathrm{~K}, 373 \mathrm{~K}, 378 \mathrm{~K}$ when the Biot number increased to $\mathrm{Bi}=10$. 
(a)

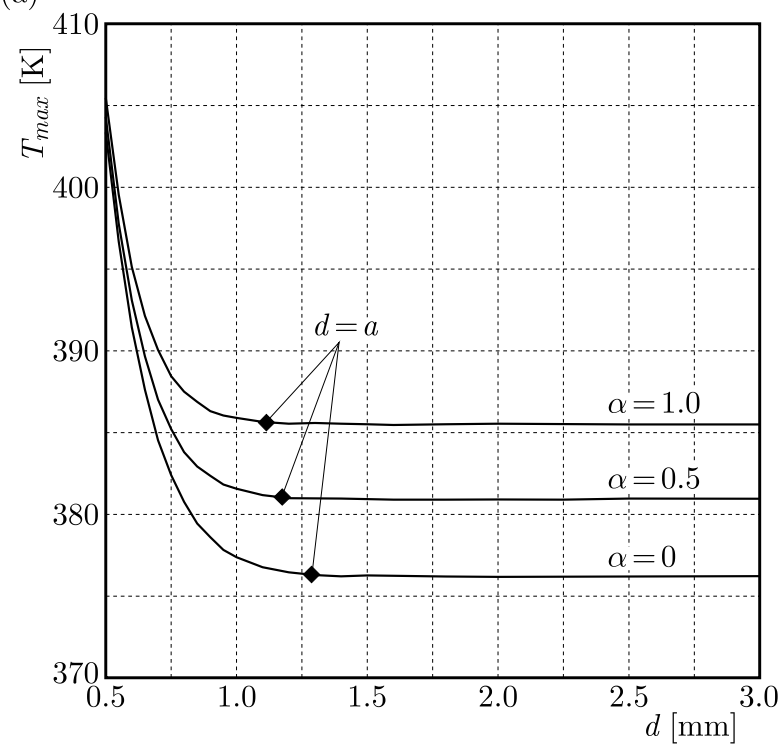

(b)

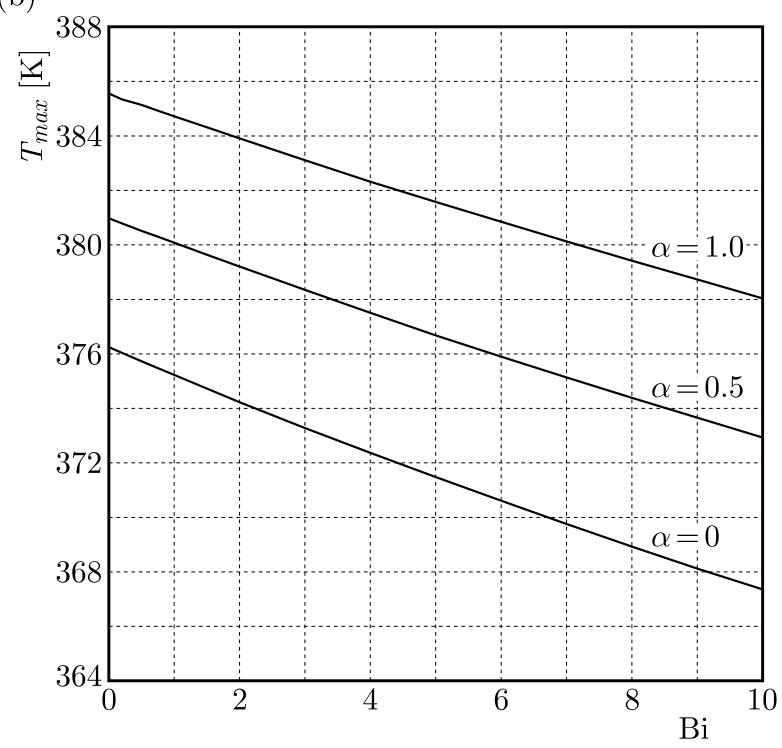

Fig. 4. Dependencies of the maximum temperature $T_{\max }(0, t)$ attained in the friction surface during sliding on the friction facing $d$ (a) and Biot numer Bi (b) for different values of $\alpha$ parameter

\section{Conclusions and remarks}

In this research paper, a new analytical solution of the thermal friction problem was presented. Based on this new solution, one can be reduce the time consumed to calculate distributions of temperature and thermal stresses in the friction surface and at any position through thickness of the friction facing of a single-plate clutch at any instant of time during the slipping process. The results showed how these distributions depend on operational parameters with a special emphasis put on the influence of thickness of frictional facings and intensity of convective heat exchange from lateral surfaces of these elements. The results of performed investigation support the thesis that in a short single engagement of the clutch system, for specified dimensions and thermophysical properties of materials, the convective cooling does not cause a significant drop in the maximum temperature. The superficial temperature obtained from the formulated model for a semi-infinite body (Kuciej and Topczewska, 2019) is almost coinciding in every instant of time. It is illustrated that thickness of the frictional facing affects the maximum temperature achieved on the heated surface when it is lower than the effective depth of heat penetration, which depends on thermal diffusivity of material and the time of slipping.

\section{References}

1. Abdullah O.I., Schlattmann J., 2012, Effect of band contact on the temperature distribution for dry friction clutch, World Academy of Science, Engineering and Technology, International Science Index, 6, 9, 150-160

2. Abdullah O.I., Schlattmann J., 2014, Computation of surface temperatures and energy dissipation in dry friction clutches for varying torque with time, International Journal of Automotive Technology, 15, 5, 733-740

3. Abdullah O.I., Akhtar M.J, Schlattmann J., 2015, Investigation of thermo-elastic behavior of multidisk clutches, Journal of Tribology, 137, 1, 011703

4. Al-Alawi A., Yousif A., Jassim M.A., 2007, An investigation into the behavior of disc brake wear, Al-Khwarizmi Engineering Journal, 3, 2, 49-66 
5. Charron F., 1943, Partage de la chaleur entre deux corps frottants, Publ. Scient. et Techn. Ministere Air, article 182

6. FAidh-Allah M.H., 2017, Numerical and finite element contact temperature analysis of friction material's type effect on a thermal transient behavior of a single-disc dry clutch, Tribologia, 1, 35-43

7. Gradshteyn I.S., Ryzhik I.M., 2007, Table of Integrals, Series, and Products, Academic press

8. Grzelczyk D., AwrejCewicz J., 2012, Modeling of thermal phenomena in a mechanical friction clutch, Machine Dynamics Research, 36, 3, 23-28

9. Hetnarski R.B., Eslami R.M., 2009, Thermal Stresses - Advanced Theory and Applications, Springer, New York

10. Kuciej M., Topczewska K., 2019, Determination of maximum temperature of the friction pad in the single-disc clutch, Materials Science, 54, 6, 819-826

11. Luikov A.V., 1968, Analytical Heat Diffusion Theory, Academic Press, New York

12. Newcomb T.P., 1960, Temperatures reached in friction clutch transmissions, Journal of Mechanical Engineering Science, 2, 4, 273-287

13. Newcomb T.P., 1961, Calculation of surface temperatures reached in clutches when the torque varies with time, Journal of Mechanical Engineering Science, 3, 4, 340-347

14. Noda N., Hetnarski R.B., Tanigawa Y., 2000, Thermal Stresses, Lastran Corp., Rochester, New York

15. Ozisık N.M., 1993, Heat Conduction, John Wiley, New York

16. Yevtushenko A., Kuciej M., Topczewska K., 2019, Effect of the temporal profile of the friction power on temperature of a pad-disc brake system, Journal of Theoretical and Applied Mechanics, 57, 2, 416-473 